\title{
Cushing's syndrome due to unilateral multiple adrenal adenomas as an extraintestinal manifestation of familial adenomatous polyposis
}

\author{
Annemieke Roos • Emma J. Groen • André P. van Beek
}

Accepted: 22 July 2008 / Published online: 22 August 2008

(C) The Author(s) 2008. This article is published with open access at Springerlink.com

To the Editor:

Familial adenomatous polyposis (FAP) is an autosomal dominantly inherited disorder that results when adenomatous polyposis coli (APC) protein function is lost due to a mutation in the APC gene on chromosome 5q21. The development of colorectal cancer stands out as the most prevalent complication, with an incidence of almost $100 \%$ by the age of 35-40 years if left untreated. Moreover, extraintestinal complications for which medical attention is essential are not rare, and estimated life time risk presumably exceeds $30 \%$. Affected individuals can develop thyroid and pancreatic cancer, hepatoblastomas, central nervous system tumors (especially medulloblastomas), and various benign tumors like adrenal adenomas, osteomas, desmoid tumors, and dental abnormalities. We report a case of Cushing's syndrome due to multiple adrenal adenomas as an extraintestinal manifestation of FAP.

A 32-year-old man was referred to the Department of Endocrinology because of ankle edema, facial plethora and spontaneous hematomas. He gained $15 \mathrm{~kg}$ during the last 4 years. His medical history was remarkable for a subtotal colectomy with ileorectal anastomosis at age 16 , as primary prophylactic treatment for FAP. Five years later, he underwent a proctectomy with an ileal pouch-anal anastomosis. Regular endoscopic follow-up according to current guidelines was performed. On physical examination, there were clear features of hypercortisolism such as a moon face, buffalo hump, enlarged fat pads that fill the supraclavicular fossae, easy bruisability of the skin, huge purple striae at

\footnotetext{
A. Roos $(\triangle) \cdot$ E. J. Groen · A. P. van Beek

Department of Endocrinology, University Medical Center

Groningen and University of Groningen,

De Brug 4.069, AA31, P. O. Box 30.001, 9700 RB Groningen,

The Netherlands

e-mail: folkinge@zonnet.nl
}

the enlarged lower abdomen, proximal muscle wasting, and hypertension. Endocrine analyses revealed an increased urinary cortisol excretion $(4,300 \mathrm{mmol} / 24 \mathrm{~h}$, reference $<270$ ), while plasma adrenocorticotropic hormone concentrations were suppressed, pointing to an adrenal cause of Cushing's syndrome. Subsequently, computed tomography revealed two tumors $(3.5 \times 3.5$ and $3.2 \times 3.0 \mathrm{~cm})$ of the left adrenal gland with benign radiological characteristics.

Adrenal masses are frequently occurring extraintestinal manifestations of FAP. They are two to four times more common in FAP than in the general population, but the clinical spectrum of these masses in FAP does not seem to be different. This can range from benign clinically silent adenomas to classical Cushing's or Conn's syndrome. Adrenocortical carcinomas and pheochromocytomas have also been described. Once a mass in the adrenals is identified, hormonal and (serial) radiological evaluation according the $\mathrm{NIH}$ conference guidelines needs to be performed. Patients should be referred to an endocrinologist. This is especially important since recent reports suggest that up to $20 \%$ of patients with an incidentally found adrenal mass have some form of subtle hormonal overproduction resulting in an increased risk for metabolic disorders and cardiovascular disease.

Our patient was cured after left sided adrenalectomy. Histologic evaluation confirmed the presence of two adenomas, both with monomorphic cellular characteristics. Osteoporosis and two impression fractures of thoracic vertebrae had developed as a consequence of the longstanding hypercortisolemia. Genetic analysis showed a mutation of the APC gene at codon 1353 (c.4057delG, p.Glu1353fsX62), predicted to result in a premature stop codon.

Open Access This article is distributed under the terms of the Creative Commons Attribution Noncommercial License which permits any noncommercial use, distribution, and reproduction in any medium, provided the original author(s) and source are credited. 